\title{
Chest Wall and Intrathoracic Desmoid Tumors: SURGICAL EXPERIENCE AND REVIEW OF THE LITERATURE
}

\author{
E. Bölke ${ }^{1 *}$, H. Krasniqi2*, G. Lammering ${ }^{3}$, R. Engers ${ }^{5}$, C. Matuschek ${ }^{1}$, S. Gripp ${ }^{1}$, P. A. Gerber ${ }^{1}$, \\ G. Fischer ${ }^{4}$, M. Peiper ${ }^{6}$, S. Shaikh 7 , W. Budach ${ }^{1}$, K. Orth ${ }^{2}$ \\ ${ }^{1}$ Department of Radiation Oncology, Heinrich-Heine-University Düsseldorf, Germany \\ ${ }^{2}$ Department of Surgery, Klinikum Emden, Germany \\ ${ }^{3}$ Maastro Clinic, Radiation Oncology, Maastricht, The Netherlands \\ ${ }^{4}$ Institute of Pathology, Wilhelmshaven, Germany, \\ ${ }^{5}$ Institute of Pathology, Heinrich-Heine-University Düsseldorf, Germany \\ ${ }^{6}$ Department of General Surgery, Heinrich-Heine-University Düsseldorf, Germany \\ ${ }^{7}$ Department fo Anesthesiology UMass Memorial Medical Center Worcester, USA
}

\begin{abstract}
Desmoid tumors are fibroblastic/myofibroblastic neoplasms, which originate from musculo-aponeurotic structures and are classified as deep fibromatoses. Despite their benign histologic appearance and lack of metastatic potential, desmoid tumors may cause aggressive local infiltrations and compression of surrounding structures. They are often associated with female gender, familial adenomatous polyposis (FAP) and sporadically may occur at sites of previous trauma, scars or irradiation. Molecular studies have demonstrated that these patients are associated with a bi-allelic APC mutation in the affected tissue. Radical tumor resection with free margins remains the first therapy of choice. In cases with anatomical or technical limitations for a wide excision, radiation therapy represents a proven and effective alternative or supplementary treatment.
\end{abstract}

Key words: Desmoid tumors, fibromatosis, extraabdominal, intrathoracic, chest wall

Abbreviations: Computed tomography scan (CT), familial adenomatous polyposis (FAP)

\section{General Description}

Desmoid tumors (fibromatosis) are histologically benign fibroblastic/myofibroblastic neoplasms, which originate from musculo-aponeurotic structures and are classified as deep fibromatoses. Desmoid tumors may arise at any site, however, the extremities, the abdomen and the retroperitoneum are the most common sites of origin. In contrast, fibromatosis of the chest wall are much less frequent. Despite their benign histologic appearance and lack of metastatic potential, desmoid tumors may cause aggressive local infiltrations and compression of surrounding structures. A high recurrence rate exists and in anatomic locations with restricted access to surgical resections, desmoid tumors can even lead to death [1, 2]. Desmoid tumors are often associated with female gender, familial ade-

*Both authors contributed equally. nomatous polyposis (FAP) and sporadically may occur at sites of previous trauma, scars or irradiation [3-6]. Even though desmoid tumors account for only $0.03 \%$ of all neoplasms, in patients with FAP, the estimated prevalence rises to approximately $13 \%[6,7]$.

\section{Histology}

Histological examinations demonstrate long fascicles of spindle cells of variable cell-density with few mitoses and the absence of atypical nucleus-separations. Characteristically, there is a diffuse cell infiltration of adjacent tissue structures. Immunohistochemically, the spindle cells are positive for vimentin, smooth muscle actin and muscle-specific actin, reflecting a fibroblastic/myofibroblastic differentiation. Recent findings revealed that virtually all deep fibromatoses exhibit somatic mutations of either the beta-catenin or adenomatous polyposis coli (APC) gene. Consequently, all of these tumors are immunohistochemically characterised by diffuse nuclear beta-catenin staining $[6,21]$.

\section{Molecular STUdies}

Notably, molecular studies have demonstrated that desmoid tumors in autosomal inherited FAP disease show clonal neoplasms arising from germline mutations or changes in the APC gene [1]. These are associated with a bi-allelic APC mutation in the affected tissue $[2,8]$. In individuals lacking germline mutations the development of desmoid tumors requires a rare combination of events, such as at least two somatic mutations in both alleles of a single tumor suppressor gene, in this case the APC gene. Correspondingly, FAP patients, exhibiting a germline mutation of the APC gene require only a single additional somatic mutation of the second allele of the APC gene for desmoid tumor development [9].

\section{TUMOR RESECTION}

To date, radical tumor resection with free margins remains the first therapy of choice. Depending on the 
tumor localisation, surgery might sometimes cause major soft tissue defects. Although abdominal wall integrity can be restored with direct sutures, reconstruction using synthetic materials is a common technique in major abdominal and/or thoracic wall defects. Greater wall defects not suitable for reconstruction by local flaps can be covered by free muscle flaps.

\section{RADIATION THERAPY}

Even though radical surgical approaches are usually favoured, radiation therapy is a suitable alternative therapeutic option. A substantial amount of evidence exists supporting the efficacy of radiation therapy for the treatment of desmoid tumors [12-16]. Radiation therapy may be used alone or in combination with surgery, either preoperatively or postoperatively.

\section{Alternative Therapeutic Options}

Other alternative therapeutic options like anti-inflammatory treatments, hormone therapy and chemotherapy have not been proven to provide any therapeutic effectiveness. Therefore these therapies are limited to patients, in whom resection is technically unfeasible, e.g. due to widespread tumor infiltration.

\section{Trans Gene Expression Therapy}

Due to the germline mutations of the APC alleles, pre-clinical studies of gene transfer for the treatment of desmoid disease in FAP have been performed [11]. However, further studies are needed to assess the clinical effects of trans gene expression therapy.

\section{Example of One Patient Studied}

The chest wall is the most common extraabdominal site for desmoid tumors [17]. However, true intrathoracic desmoid tumors are extremely rare with most cases representing intrathoracic extension of chest wall tumors, like the case presented below.

For this report, a review of the literature was undertaken concerning chest wall and intrathoracic desmoid tumors and we report on our surgical experi- ence with one case of an extreme intrathoracic extension of a chest wall tumor:

A 17-year-old woman from Kosovo visiting her relatives in Germany was admitted to our hospital with chronic respiratory insufficency. She reported about a "tumor" in her right hemithorax which had been treated previously with "alternative medicine" for the last 5 years. The CT-scan of the chest revealed complete compression of her right lung by the tumor-mass and a consecutive dislocation of the mediastinum (panel a, b). Histopathological evaluation of biopsies revealed a fibromatosis (panel e, $\mathrm{f}, \mathrm{g}$ ).

Intraoperatively, it became apparant that the mass had originated from the intercostal muscle of the right upper thorax and filled up the complete hemithorax. Nevertheless, it was technically feasible to completely remove the tumor including a resection of two ribs (panel c, d). Consecutively, the small poorly developed right lung was mobilised and the thoracic wall defect was reconstructed using synthetic materials (polypropylene net) in a common technique. The postoperative follow up was uneventful and the patient was discharged home on the 14th postoperative day.

Histological examination of the resected specimen revealed a tumor which consisted of elongated, slender, spindle-shaped cells of uniform appearance. These cells were embedded in abundant collagen with little or no cell-to-cell contact. Cellular boundaries were poorly defined. Nuclei were small, pale-staining and sharply defined with only minute nucleoli (panel f). The immunohistochemistry revealed a minority of spindle shaped tumor cells, expressing the proliferative Ki 67 antigen (panel g). Based on these characteristics the diagnosis of an extra-abdominal fibromatosis (desmoid tumor) was made.

\section{DISCUSSION}

Desmoid tumors involving the chest and adjacent structures are locally aggressive tumors with a high recurrence rate. In the literature, recurrences are strongly associated with positive margins, which underlines the need for wide radical resection of margins whenever possible [17]. Desmoid tumors have a remarkable
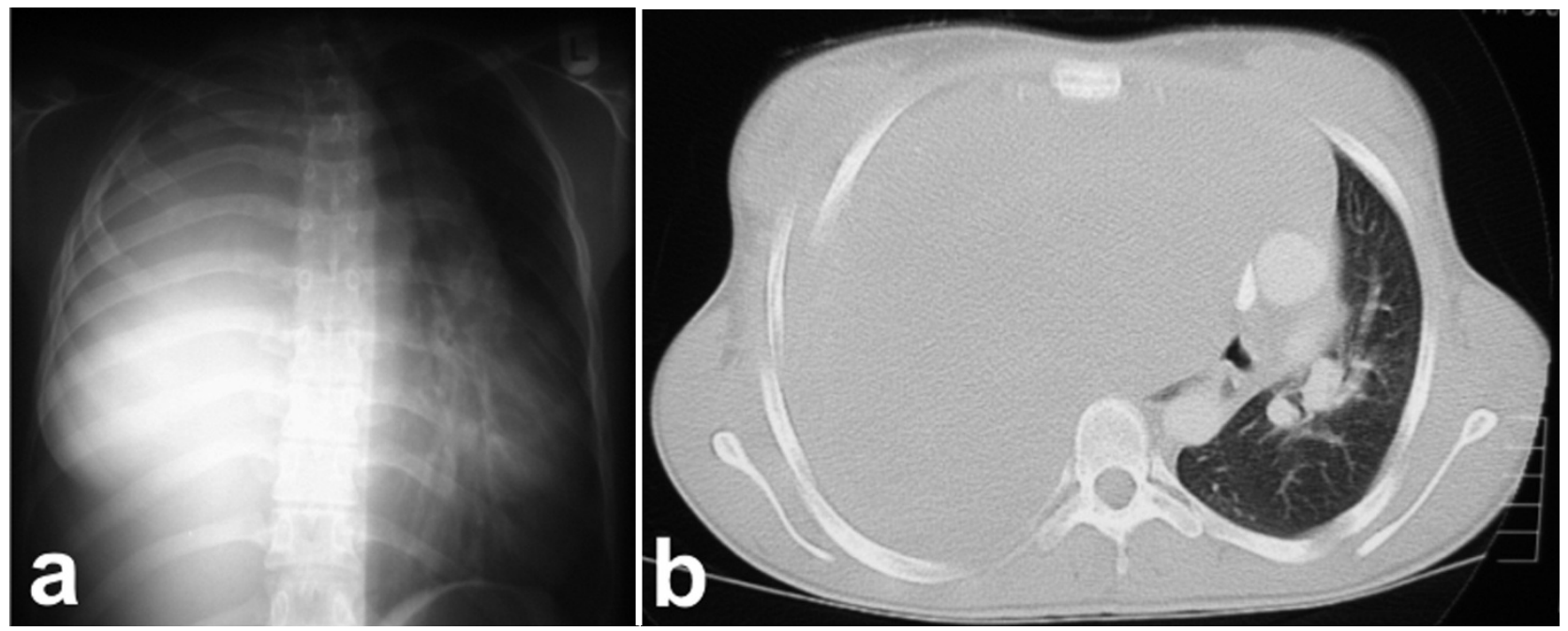

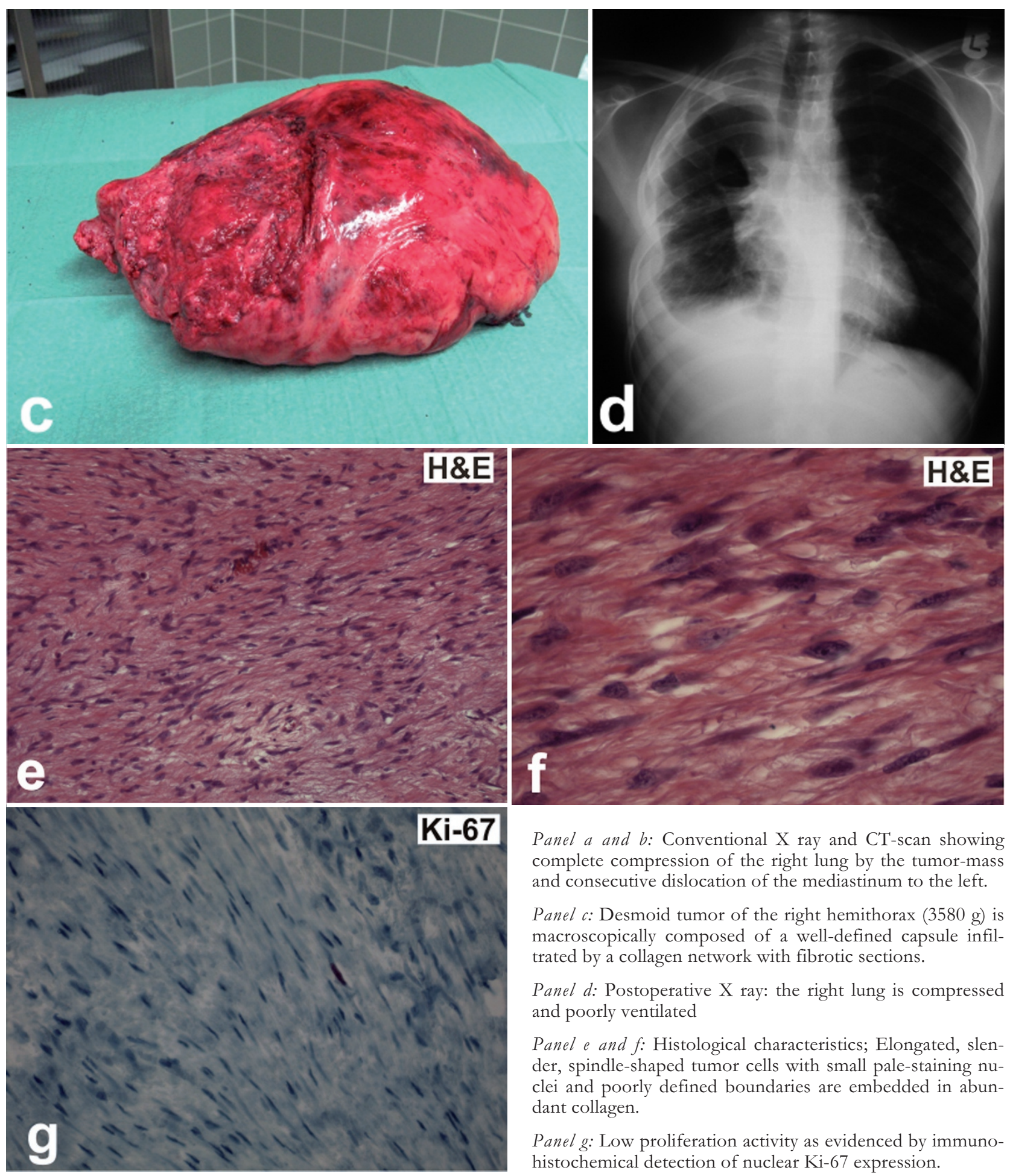

Panel $a$ and $b$ : Conventional $\mathrm{X}$ ray and CT-scan showing complete compression of the right lung by the tumor-mass and consecutive dislocation of the mediastinum to the left.

Panel c: Desmoid tumor of the right hemithorax (3580 g) is macroscopically composed of a well-defined capsule infiltrated by a collagen network with fibrotic sections.

Panel d: Postoperative $\mathrm{X}$ ray: the right lung is compressed and poorly ventilated

Panel $e$ and $f$ : Histological characteristics; Elongated, slender, spindle-shaped tumor cells with small pale-staining nuclei and poorly defined boundaries are embedded in abundant collagen.

Panel g: Low proliferation activity as evidenced by immunohistochemical detection of nuclear Ki-67 expression.

tendency to infiltrate into surrounding structures [19]. For this reason, wide radical resection has been considered essential for successful management [20]. However, obtaining a negative margin involving the chest region often represents a therapeutic challenge for the surgeon. Unlike superficial chest wall desmoid tumors which will create a palpable mass, intrathoracic tumors will not cause symptoms until they grow large enough to locally invade surrounding structures or compress pulmonary parenchyma, like the case presented herein. Thus, wide local excision is often impossible because of surrounding vascular and neur- al structures. Therefore, it may be advisable to consider radiation therapy as an alternative or concomitant treatment for desmoid tumors. The efficacy of radiation therapy for the treatment of desmoid tumors is supported by substantial amount of evidence [10, 1216]. For patients with positive margins or residual disease after surgery, adjuvant radiation therapy in combination with surgery results in equivalent local control to surgery with negative margins. Thus, for patients who are not surgical candidates or who refuse surgery, radiation therapy alone results in durable local control rates of 70 to $80 \%[10,12-16]$. Disease re- 
gression may take some time after radiation therapy, however, long-term local control rates are excellent. Normal tissue toxicity and potential late radiation effects, including secondary malignancies, are important considerations in the treatment of otherwise healthy, often young patients. However, the typical radiation dose necessary for the treatment ( $50 \mathrm{~Gy}$ ) does not exceed bowel tolerance and is associated with a low risk of complications [15]. Furthermore, the use of modern, conformal radiation treatment planning techniques allows for a minimization of normal tissue dose. When considering disease progression that could result in significant morbidity and mortality, the potential benefits of radiation therapy clearly outweigh the risks.

In conclusion, primary radical resection with negative margins remains the principal determinant of outcome for intrathoracic or chest wall desmoid tumors. Positive margins after surgery reflect a high risk for local recurrence. Thus, in cases with anatomical or technical limitations for a wide excision, it may be advisable to consider radiation therapy as an alternative or concomitant treatment. Radiation therapy represents a proven and effective therapy for this disorder.

\section{REFERENCES}

1. Brueckl WM, Ballhausen WG, Förtsch T, Günther K, Fiedler W, Gentner B, et al. Genetic testing for germline mutations of the APC gene in patients with apparently sporadic desmoid tumors with a family history of colorectal carcinoma. Dis Colon Rectum 2005; 48: 1275-1281

2. Sturt NJ, Gallagher MC, Bassett P, Philp CR, Neale KF, Tomlinson IP, et al. Evidence for genetic predisposition to desmoid tumours in familial adenomatous polyposis independent of the germline APC mutation. Gut 2004; 53: 1832- 1836

3. Raynham WH, Louw JH. Desmoid tumours in familial polyposis of the colon. S Afr J Surg 1971; 9: 133-140

4. Lopez R, Kemalyan N, Moseley HS, Dennis D, Vetto RM. Problems in diagnosis and management of desmoid tumors. Am J Surg 1990; 159: 450-453

5. Gaches C, Burke J. Desmoid tumour (fibroma of the abdominal wall) occurring in siblings. Br J Surg 1971; 58: 495-498

6. Lee JC, Thomas JM, Phillips S, Fisher C, Moskovic E. Aggressive fibromatosis: MRI features with pathologic correlation. AJR Am J Roentgenol 2006; 186: 247-254

7. Shields CJ, Winter DC, Kirwan WO, Redmond HP. Desmoid tumours. Eur J Surg Oncol 2001; 27: 701-706

8. Klemmer S, Pascoe L, DeCosse J. Occurrence of desmoids in patients with familial adenomatous polyposis of the colon. Am J Med Genet 1987; 28: 385-392

9. Caspari R, Olschwang S, Friedl W, Mandl M, Boisson C, Böker T, et al. Familial adenomatous polyposis: desmoid tumours and lack of ophthalmic lesions (CHRPE) associated with APC mutations beyond codon 1444. Hum Mol Genet 1995; 4: 337-340
10. Goy BW, Lee SP, Eilber F, Dorey F, Eckardt J, Fu YS, Juillard GJ, Selch MT: The role of adjuvant radiotherapy in the treatment of resectable desmoid tumors. Int J Radiat Oncol Biol Phys 1997; 39: 659-665.

11. Bright-Thomas RM, Agrawal A, Hargest R: Preclinical studies of gene transfer for the treatment of desmoid disease in familial adenomatous polyposis. Br J Surg 2002; 89:1563-1569

12. Micke O, Seegenschmiedt MH, German Cooperative group on Radiotherapy for Benign Diseases. Radiation Therapy for Aggressive Fibromatosis (Desmoid Tumors): Results of a National Pattern of Care Study. Int Rad Onc Biol Phys 2005;61:882-891.

13. Ballo MT, Zagars GK, Pollack A et al. Desmoid tumor: Prognostic factors and outcome after surgery, radiation therapy, or combined surgery and radiation therapy. J Clin Oncol 1999;17:158-167.

14. Nuyttens JJ, Rust PF, Thomas CR Jr, et al.: Surgery versus radiation therapy for patients with aggressive fibromatosis or desmoid tumors: A comparative review of 22 articles. Cancer 2000;88:1517-1523.

15. Ballo MT, Zagars GK, Pollack A: Radiation therapy in the management of desmoid tumors. In J Radiat Oncol Phys 1998;42:1007-1014.

16. Guadagnolo BA, Zagars GK, Ballo MT. Long-term outcomes for desmoid tumors treated with radiation therapy. Int J Rad Oncol Biol Phys 2008;71:441-447

17. Abbas AE, Deschamps C, Cassivi SD, Nicols III FC, Allen MS, Schleck CD, Pairlero PC. Chest wall desmoid tumors: results of surgical intervention. Ann Thorac Surg 2004;78:1219-1223.

18. Meyerson SL, Dámico TA. Intrathoracic desmoid tumor: brief report and review of the literature. J Thorac Oncol 2008;3:656-659.

19. Allen PJ, Shriver CD. Desmoid tumors of the chest wall. Semin Thorac Cardiovasc Surg. 1999;11:264-9.

20. Pairolero PC, Arnold PG. Chest wall tumors. Experience with 100 consecutive patients. J Thorac Cardiovasc Surg. 1985;90:367-72.

21. Desmoid tumor: a disease opportune for molecular insights. Kotiligam D, Lazar AJ, Pollock RE, Lev D. Histol Histopathol. 2008 Jan;23(1):117-26. Review

Received: March 9, 2009 / Accepted: April 14, 2009

Address for correspondence:

Professor Dr. Wilfried Budach

Department of Radiation Oncology

University Hospital Düsseldorf

Moorenstr. 5

40225 Düsseldorf

Germany

Phone: $\quad+49211 / 8117991$

Fax: +49 211/8118051

E-mail: wilfried.budach@uni-duesseldorf.de 